ARTICLE

DOI: $10.1038 / s 41467-017-00266-4$

\title{
Solution nuclear magnetic resonance spectroscopy on a nanostructured diamond chip
}

\author{
P. Kehayias (1) 1,2, A. Jarmola 3,4, N. Mosavian², I. Fescenko², F.M. Benito (1) 2, A. Laraoui (D) 2, J. Smits², \\ L. Bougas ${ }^{5}$, D. Budker (1) 3,4,6, A. Neumann², S.R.J. Brueck² \& V.M. Acosta (1) ${ }^{2}$
}

Sensors using nitrogen-vacancy centers in diamond are a promising tool for small-volume nuclear magnetic resonance (NMR) spectroscopy, but the limited sensitivity remains a challenge. Here we show nearly two orders of magnitude improvement in concentration sensitivity over previous nitrogen-vacancy and picoliter NMR studies. We demonstrate NMR spectroscopy of picoliter-volume solutions using a nanostructured diamond chip with dense, high-aspect-ratio nanogratings, enhancing the surface area by 15 times. The nanograting sidewalls are doped with nitrogen-vacancies located a few nanometers from the diamond surface to detect the NMR spectrum of roughly $1 \mathrm{pl}$ of fluid lying within adjacent nanograting grooves. We perform ${ }^{1} \mathrm{H}$ and ${ }^{19} \mathrm{~F}$ nuclear magnetic resonance spectroscopy at room temperature in magnetic fields below $50 \mathrm{mT}$. Using a solution of CsF in glycerol, we determine that $4 \pm 2 \times 10^{12}{ }^{19} \mathrm{~F}$ spins in a $1 \mathrm{pl}$ volume can be detected with a signal-to-noise ratio of 3 in $1 \mathrm{~s}$ of integration.

\footnotetext{
${ }^{1}$ Department of Physics, Harvard University, Cambridge, 02138 MA, USA. ${ }^{2}$ Center for High Technology Materials, Department of Physics and Astronomy, University of New Mexico, Albuquerque, 87106 NM, USA. ${ }^{3}$ ODMR Technologies Inc., El Cerrito, 94530 CA, USA. ${ }^{4}$ Department of Physics, University of California-Berkeley, Berkeley, 94720 CA, USA. ${ }^{5}$ Johannes Gutenberg Universität Mainz, 55128 Mainz, Germany. ${ }^{6}$ Helmholtz Institut Mainz, 55099 Mainz, Germany. P. Kehayias and A. Jarmola contributed equally to this work. Correspondence and requests for materials should be addressed to A.J.(email: andrey.jarmola@odmrtechnologies.com) or to V.M.A.(email: vmacosta@unm.edu)
} 
$\mathrm{N}$ uclear magnetic resonance (NMR) spectroscopy is an invaluable analytical tool for determining the composition, structure, and function of complex molecules. However, conventional high-field NMR spectrometers have drawbacks. Owing to their reliance on cryogenic magnets, the best NMR spectrometers are massive, immobile, and expensive. Often they require relatively large quantities of analyte and large $(\gtrsim 10 \mu \mathrm{l})$ sample volumes to overcome fundamental sensitivity constraints from inductive detection and low thermal polarization. This limits NMR use in sample-limited analysis and high-throughput screening, where a parallel microfluidic platform would be preferable ${ }^{1-3}$. Microcoil NMR ${ }^{4-8}$ can lower the required analyte volume to $\gtrsim 1 \mathrm{nl}$, but still requires a large magnetic field for sensitive spectroscopy. Nuclear hyperpolarization methods and alternative magnetometry technologies 9,10 enable certain experiments at lower fields, but there is still no platform that combines the high sensitivity and sub-nl analyte volume necessary for operation in microfluidic assays.

Recently, a new technique has emerged for NMR spectroscopy at the nanometer scale based on optical detection of the electron spin resonances of negatively charged nitrogen-vacancy (NV) color centers in diamond ${ }^{11-18}$. The technique relies on statistical nuclear polarization, which is substantially larger than thermal polarization for nanoscale sensing volumes ${ }^{19}$. Combined with non-inductive optical detection, this renders the NV NMR sensitivity independent of temperature and magnetic field. Previous lines of work used single NV centers or an NV ensemble $e^{11-17}$ to detect NMR of nuclei in liquids and thin films across a flat sample-sensor interface. While this was a significant scientific breakthrough, the long measurement time (hours to days) is a liability for many applications.

In this work we demonstrate picoliter ( $\mathrm{pl}$ ) solution NMR using a nanostructured diamond chip. The NMR detection sensitivity depends on the number of NV centers that are located close enough to the diamond surface to sense external spins. To increase this number, the diamond surface is lithographically structured with dense, high-aspect-ratio nanogratings to enhance the sensor-analyte contact area by $\gtrsim 15 \times$. The nanostructure sidewalls are then doped with a high density of NV centers. The result is tens of millions of NV centers located close enough to the diamond surface $(5-20 \mathrm{~nm})$ to detect the NMR spectrum from $\sim 1 \mathrm{pl}$ of fluid lying within the adjacent nanograting grooves. This leads to a corresponding boost in fluorescence signal and reduction in acquisition time. With further improvements in spectral resolution, this platform could enable a wide variety of applications in biochemistry, including pharmacodynamic studies of metabolites and natural products and high-throughput screening for drug discovery.

\section{Results}

Statistical polarization and thermal polarization. NMR is a powerful analytical technique for non-destructive molecular structure elucidation, but its detection sensitivity is orders of magnitude worse than other analytical chemistry techniques such as mass spectrometry or fluorescence labeling methods ${ }^{5}$. The sensitivity is limited by the small nuclear magnetization. At the highest magnetic field available, $B_{0}=24 \mathrm{~T}$, the room-temperature ${ }^{1} \mathrm{H}$ thermal polarization is just $10^{-4} 20$. The sensitivity is further limited by the use of inductive detection, which leads to a frequency-dependent signal-to-noise ratio. One potential remedy is to increase the magnetic field, but despite steady improvements in magnet technology, the signal strength has improved by less than a factor of two over the last 20 years ${ }^{21}$.

Alternative NMR techniques seek to increase nuclear polarization and/or improve signal-to-noise without relying on increasing a

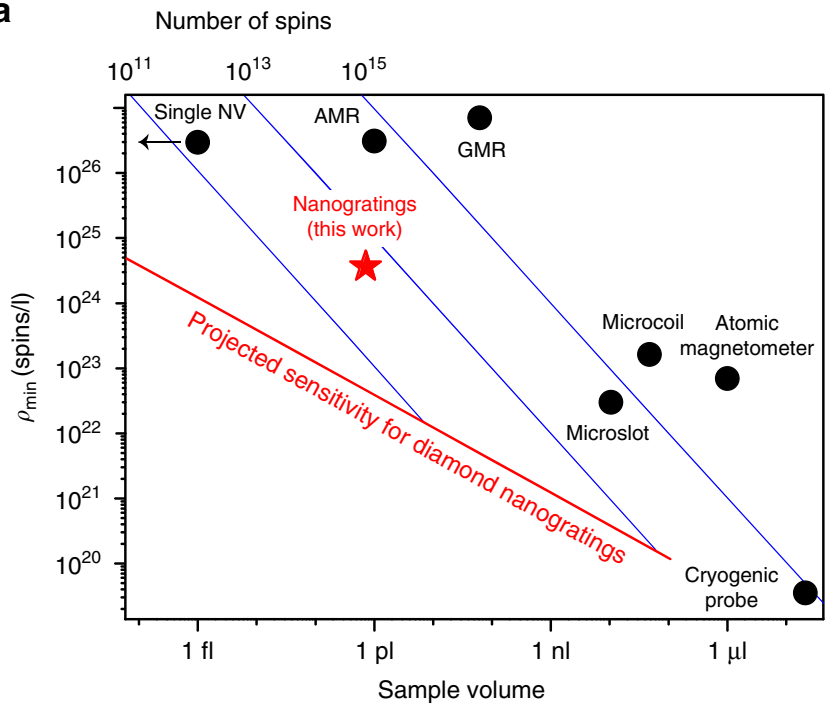

b

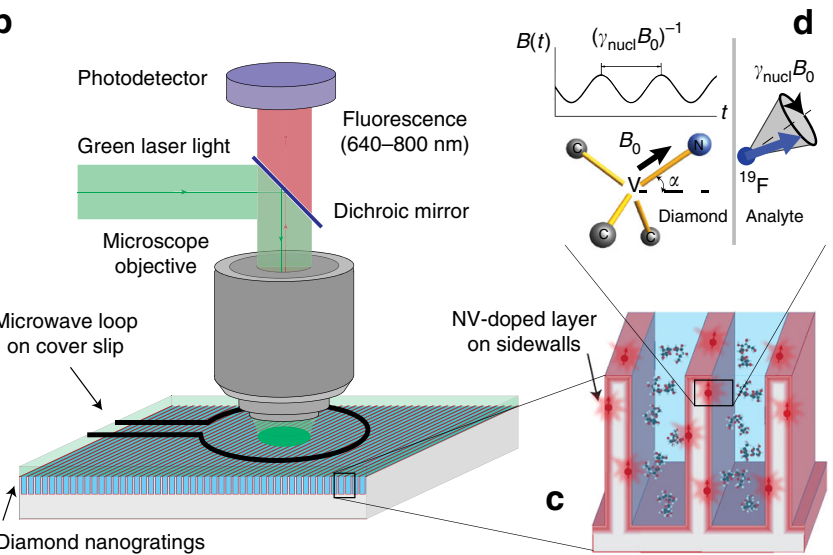

Fig. 1 Picoliter NMR. a Overview of ambient-temperature NMR techniques for small volumes. Points represent experimental values for minimum-detectable nuclear spin concentration in $1 \mathrm{~s}$ with $\mathrm{SNR}=3$ for different techniques: microslot ${ }^{7}$, microcoil ${ }^{8}$, cryogenic probes ${ }^{22}$, atomic vapor magnetometers ${ }^{25}$, giant magnetoresistance (GMR) sensors ${ }^{23}$, anistropic magnetoresistance (AMR) sensors ${ }^{24}$, single NV centers ${ }^{14}$, and NV-doped nanogratings (this work). The solid red line is the projected sensitivity for diamond nanogratings (Eq. (1)), exhibiting volume ${ }^{-1 / 2}$ scaling (see Supplementary Note 1). Solid blue lines indicate constant numbers of spins. $\mathbf{b}$ Epifluorescence diamond NMR set-up. $\mathbf{c}$ The sensor region consists of dense, high aspect-ratio diamond nanogratings fabricated via interferometric lithography and doped with NV centers. d Experimental geometry. The analyte's precessing nuclear statistical polarization produces an oscillating magnetic field, which is sensed by adjacent near-surface NV centers

$B_{0}$. The latter can be accomplished by cryogenically cooling the inductive probe ${ }^{22}$ or switching to non-inductive detection modalities, including giant magnetoresistance sensors ${ }^{23}$, anistropic magnetoresistance sensors ${ }^{24}$ or atomic magnetometers ${ }^{25}$. The present NV-based NMR approach uses non-inductive detection to sense the statistical nuclear polarization, an effect that arises from imperfect cancellation of the net magnetization from an ensemble of randomly oriented spins ${ }^{11}$ 12. Statistical polarization is larger than thermal polarization for sufficiently small numbers of nuclear spins (see Supplementary Note 1) and makes the NV NMR sensitivity independent of sample temperature and $B_{0}$. Figure la summarizes existing NMR techniques for small sample volumes. The NMR 

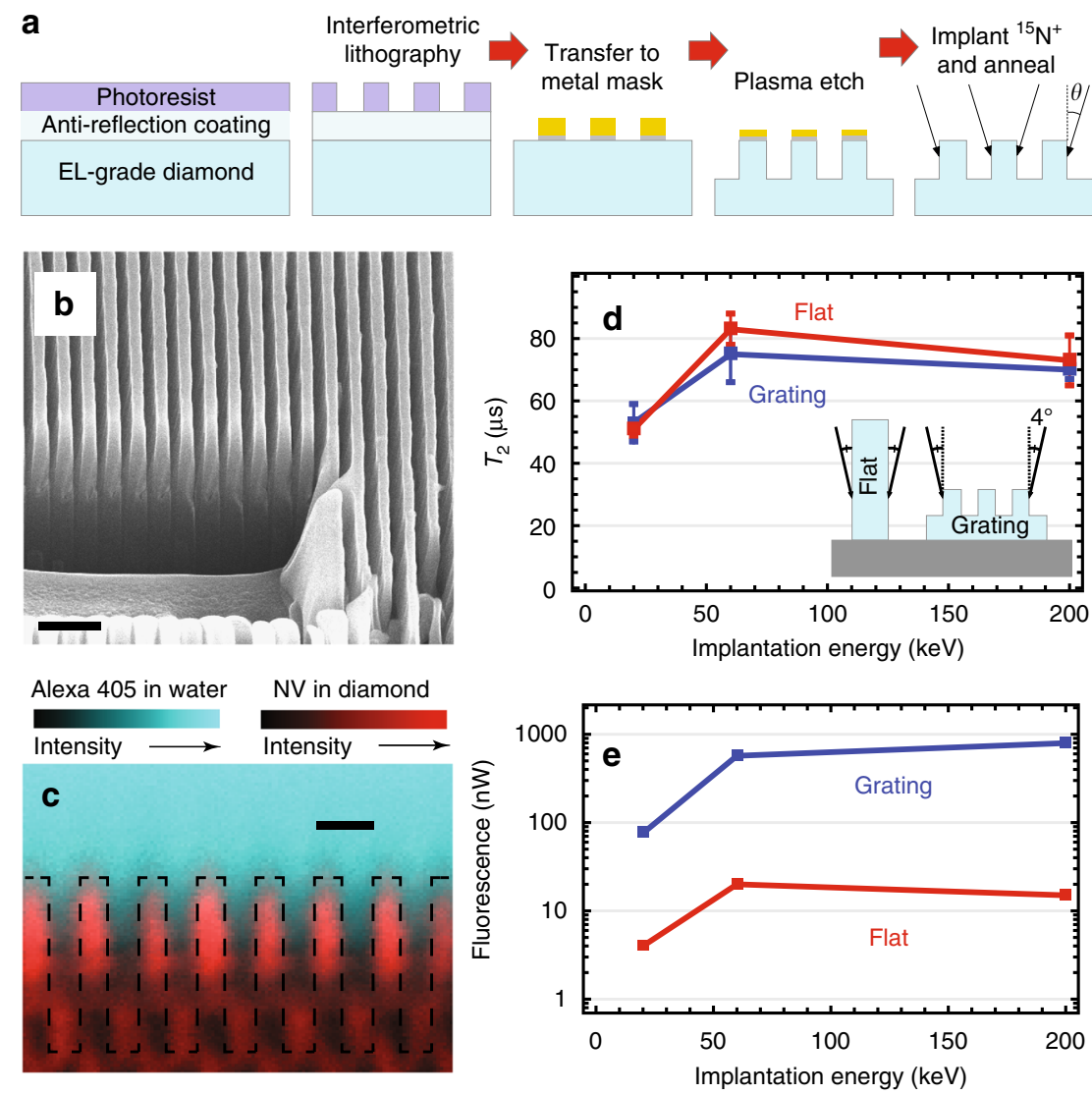

Fig. 2 Diamond nanogratings. a Schematic of large-area nanofabrication process. b Scanning electron micrograph of $400 \mathrm{~nm}$ pitch diamond nanogratings. Focused ion beam etching prior to imaging enabled visualization of the nanogratings' cross-section. Scale bar is $1 \mu \mathrm{m}$. c Confocal microscopy images reveal that fluorescence from dye-stained water originates from areas inside the nanograting grooves, confirming wetting. Dashed lines represent the estimated diamond-water boundary. Scale bar is $500 \mathrm{~nm}$. d Comparison of $T_{2}$, measured with the XY8-22 protocol, and e fluorescence intensity between flat and nanograting chips implanted at similar conditions. $T_{2}$ can surpass $\sim 100 \mu$ s with sufficient decoupling $\pi$-pulses (see Supplementary Note 2 ). Error bars in d represent standard error of the mean

sensitivity is characterized by the minimum spin concentration detectable in $1 \mathrm{~s}$ at room temperature with signal-to-noise ratio (SNR) of 3.

For NV NMR the minimum detectable spin concentration $\rho_{\text {min }}$ for $\mathrm{SNR}=3$ in $1 \mathrm{~s}$ is (see Supplementary Note 1 ):

$$
\rho_{\text {min }}=\frac{3}{P(\alpha)\left(\mu_{0} \hbar \gamma_{\mathrm{NV}} \gamma_{\mathrm{nucl}}\right)^{2}} \times \frac{d_{\mathrm{NV}}^{3}}{T_{\mathrm{tot}}^{2} C \sqrt{\eta N_{\mathrm{NV}} N_{\mathrm{r}}}},
$$

where $P(\alpha)=\left\{\pi\left[8-3(\sin \alpha)^{4}\right]\right\} / 128$ is a geometric factor that comes from the angle $\alpha$ the $\mathrm{N}-\mathrm{V}$ axis makes with the diamond surface normal (Fig. 1c, d), $\mu_{0}=4 \pi \times 10^{-7} \mathrm{~m} \mathrm{~T} / \mathrm{A}$ is the vacuum permeability, $\hbar=1.055 \times 10^{-34} \mathrm{~J} \cdot \mathrm{s}$ is the reduced Planck constant, $\gamma_{\mathrm{NV}}=28.03 \mathrm{GHz} / \mathrm{T}$ is the $\mathrm{NV}$ gyromagnetic ratio, $\gamma_{\text {nucl }}$ is the nuclear gyromagnetic ratio $\left(42.58 \mathrm{MHz} / \mathrm{T}\right.$ for ${ }^{1} \mathrm{H}$ and $40.08 \mathrm{MHz} / \mathrm{T}$ for $\left.{ }^{19} \mathrm{~F}\right), d_{\mathrm{NV}}$ is the characteristic NV depth below the diamond surface, $T_{\text {tot }}$ is the NV phase accumulation time during a single XY8-N pulse sequence (Fig. 3a), $C$ is the $\mathrm{NV}$ fluorescence-detected spin contrast, $N_{\mathrm{NV}}$ is the number of near-surface $\mathrm{NV}$ centers in the sensing area, $\eta$ is the mean number of photons collected per NV per readout $(\eta<1)$, and $N_{\mathrm{r}}$ is the number of readouts per second.

Since $\rho_{\min } \propto 1 / \sqrt{\eta N_{\mathrm{NV}}}$, the sensitivity can be improved by increasing the sensor surface area, boosting $N_{\mathrm{NV}}$ for a constant laser spot size. To this end, we nanostructured the diamond surface with high-aspect-ratio nanogratings and doped the sidewalls with a high density of NV centers.
Diamond chip fabrication and characterization. Nanogratings were etched into the surface of electronic-grade [100]-polished diamond chips using optical interferometric lithography ${ }^{26,27}$ and diamond plasma etching (see Supplementary Note 2 and refs. $\left.{ }^{28}, 29\right)$, as shown in Fig. 2a. The nanogratings have a $400 \mathrm{~nm}$ pitch and a depth up to $3 \mu \mathrm{m}$ (Fig. 2b). By varying the resist, postbake, and development conditions, duty cycles of $20-80 \%$ are achievable. The nanograting sidewalls were doped with NV centers by implanting ${ }^{15} \mathrm{~N}^{+}$ions at angles $\theta= \pm 4^{\circ}$ relative to the substrate surface normal. The angles were chosen to ensure that an entire 3 - $\mu \mathrm{m}$-tall sidewall would be doped. Each chip was implanted with either 20, 60, or $200 \mathrm{keV}$ ion energy, corresponding to $\sim 5,10$, and $20 \mathrm{~nm}$ simulated NV depths, respectively (see Supplementary Note 2 for doses and other information). After implantation, the chips were annealed in vacuum at $800-1100^{\circ} \mathrm{C}$ to form NV centers ${ }^{30}$. We investigated the analyte/nanograting adhesion (wetting) with confocal microscopy. Water stained with Alexa 405 dye was dispersed on top of an NV-doped nanograting chip. Afterwards, fluorescence from the NV centers $(650-800 \mathrm{~nm})$ and dye-stained water $(425-500 \mathrm{~nm})$ was simultaneously imaged. We confirmed that the nanogratings were wetting by observing Alexa 405 fluorescence from areas inside the nanograting grooves (Fig. 2c).

Optical NMR detection was performed with a custom-built epifluorescence microscope with pulsed laser and microwave interrogation, shown in Fig. 1b (see Supplementary Note 2). Using $\sim 140 \mathrm{~mW}$ of $520 \mathrm{~nm}$ laser light over a $25-\mu \mathrm{m}$-diameter 
a

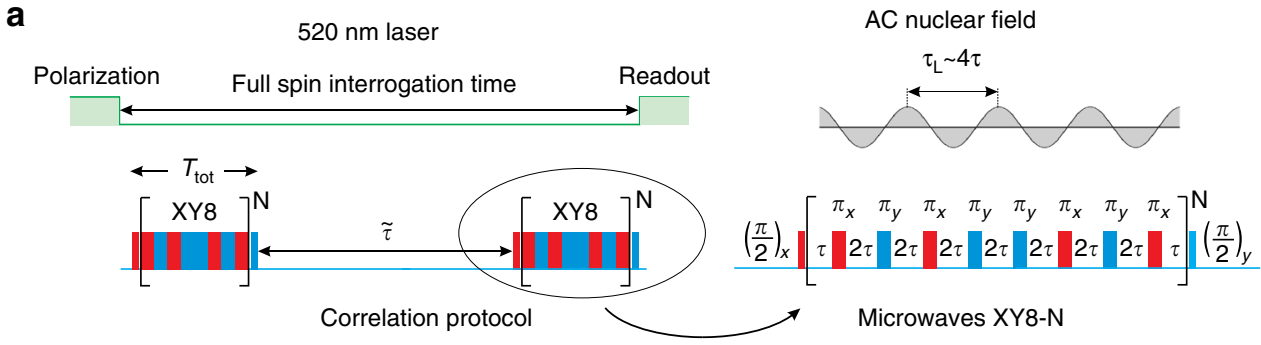

b

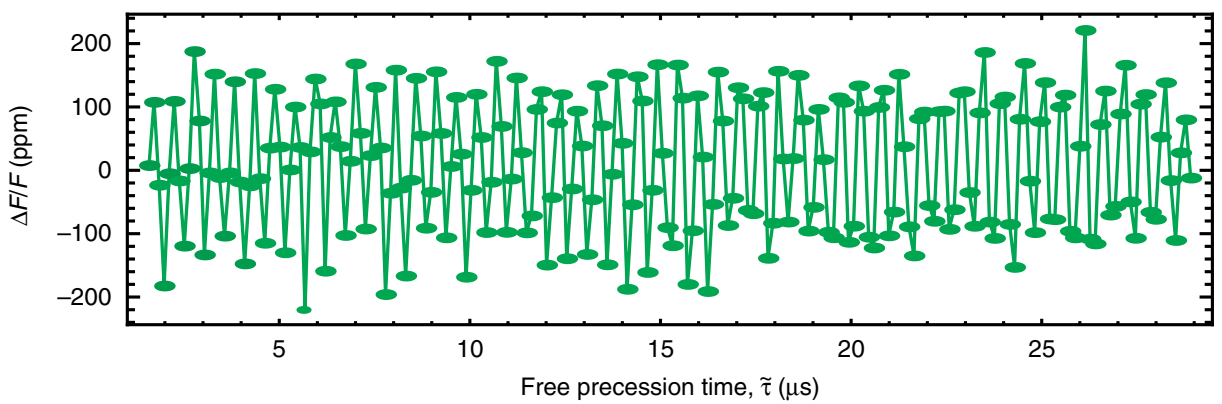

C

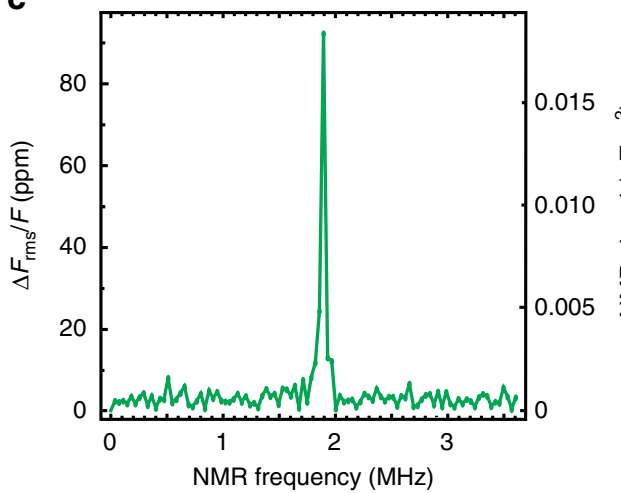

d

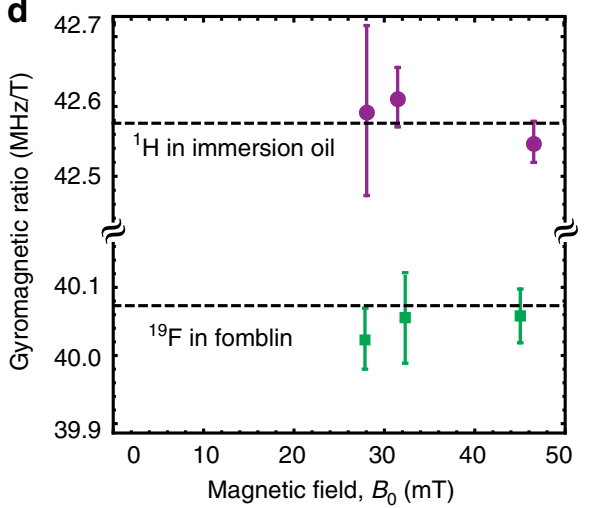

Fig. 3 Nanoscale NMR. a Sensing protocols: optical pulses are used to pump and probe NV spin state via the spin-dependent fluorescence; microwave multipulse sequences are applied between optical pump and probe pulses. Red and blue color indicates different microwave phases, which are shifted relative to each other by $90^{\circ}$. NV centers are resonantly tuned to detect a particular nuclear species by setting $4 \tau=\tau_{L}$, where $2 \tau$ is the separation between $\pi$-pulses and $\tau_{\mathrm{L}}$ is the nuclear precession period. In order to reject common-mode noise the sequences are repeated with the phase of the last $\pi / 2$-pulse shifted by $180^{\circ}$. The resulting signals are then subtracted and normalized to give the measurement results. $\mathbf{b}$ Time-domain NMR signal for ${ }^{19} \mathrm{~F}$ nuclei in Fomblin ${ }^{\circledR}$ oil taken using XY8-13 correlation sequence. $\mathbf{c}^{19} \mathrm{~F}$ frequency-domain NMR signal at $B_{0}=47.1 \mathrm{mT}$ obtained by Fourier transform of the data in b. d Measured ${ }^{1} \mathrm{H}$ and ${ }^{19} \mathrm{~F}$ gyromagnetic ratios at different $B_{0}$ values. Dashed lines are literature values ${ }^{51}$. Error bars represent standard error of the mean

spot fluorescence from $\gtrsim 10$ million NV centers adjacent to $\sim 1 \mathrm{pl}$ of analyte was detected. The analyte volume reported here is the physical volume the solution occupies within the laser spot, which is given by the product of the grating height $(\sim 3 \mu \mathrm{m})$, grating duty cycle $(\sim 0.5)$, and laser spot area $\pi \times(25 \mu \mathrm{m} / 2)^{2}$, or $0.7 \mathrm{pl}$. A static magnetic field $B_{0}=20-50 \mathrm{mT}$ was applied along one of the four $\mathrm{NV}$ axes with a permanent magnet, and the aligned $\mathrm{NV}$ sub-ensemble was optically interrogated using resonant microwaves (10-15 MHz Rabi frequency) delivered by a copper loop fabricated on a cover slip.

To assess the anticipated improvement of the nanograting chips, the nanograting sidewalls and the flat surfaces of unstructured diamond chips were doped at similar conditions to have the same nitrogen density (Fig. 2d, inset). Figure $2 \mathrm{~d}$, e compares the NV transverse spin coherence time $T_{2}$ (measured with the XY8-22 pulse sequence ${ }^{31}$ ) and fluorescence intensity for flat and nanograting chips implanted at different energies. While $\mathrm{T}_{2}$ for flat and nanograting chips is approximately the same (and is similar to $T_{2}$ for normal-incidence high-dose implantation ${ }^{13}$ ), the fluorescence intensity is $20-50$ times brighter for nanograting chips. We attribute this to the $2 / \tan 4^{\circ}=28$ times higher effective dose captured by the nanograting chip and improved collection efficiency from nanostructures ${ }^{32}$. These results highlight the advantage of nanogratings in NMR sensitivity, since fluorescence intensity is proportional to $\eta N_{\mathrm{NV}}$, whereas $\rho_{\text {min }} \propto 1 / \sqrt{\eta N_{\mathrm{NV}}}$. The nanostructuring process does not negatively affect other parameters in Eq. (1). The fluorescence contrast of Rabi oscillations was $2.7 \pm 0.8 \%$ for all studied diamond chips independent of nanofabrication or doping parameters.

NV NMR spectroscopy. We used a correlation spectroscopy pulse sequence for NV NMR detection of external solutions ${ }^{33}$, as described in Fig. 3a. This sequence correlates the nuclear magnetic fields at different points in time, encoding this information in the NV spin state and corresponding fluorescence intensity. Specifically, we used two XY8-N pulse trains, both tuned to the target nuclear Larmor frequency and separated by a variable delay $\tilde{\tau}$. As $\tilde{\tau}$ is swept, the relative $\mathrm{NV}$ fluorescence intensity, $\Delta F / F$, oscillates at the nuclear Larmor frequency, analogous to a nuclear free induction decay (FID). The Fourier transform of this FID-like signal reveals the NMR spectrum, from which we extract the spin density. 
a

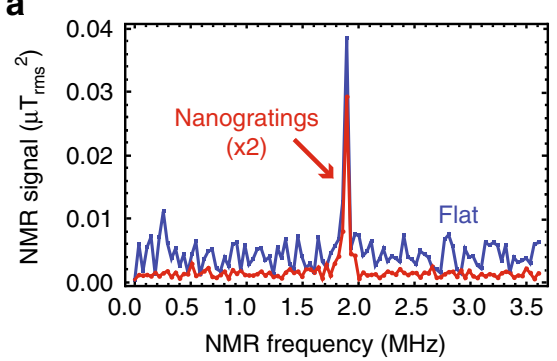

b

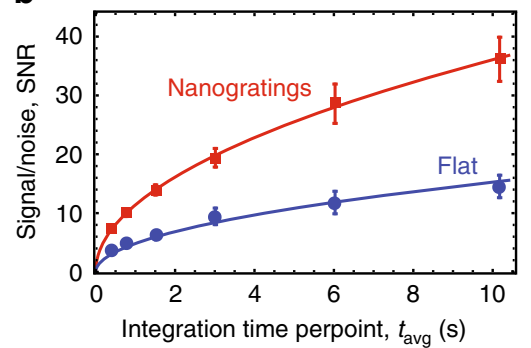

Fig. 4 NV NMR sensitivity characterization. ${ }^{19} \mathrm{~F}$ NMR signal from Fomblin ${ }^{\circledR}$ oil at $B_{0}=47.1 \mathrm{mT}$ for flat and nanograting sensors implanted at $20 \mathrm{keV}$ using XY8-13 correlation sequence. $\mathbf{b}$ NMR signal-to-noise ratio as a function of averaging time. Fits to the function SNR $=\alpha \sqrt{t_{\text {avg }}}$ give good agreement for both sensors (solid lines). The coefficient $\alpha$ was 2.4 times larger for the nanograting chip. Error bars represent standard error of the mean

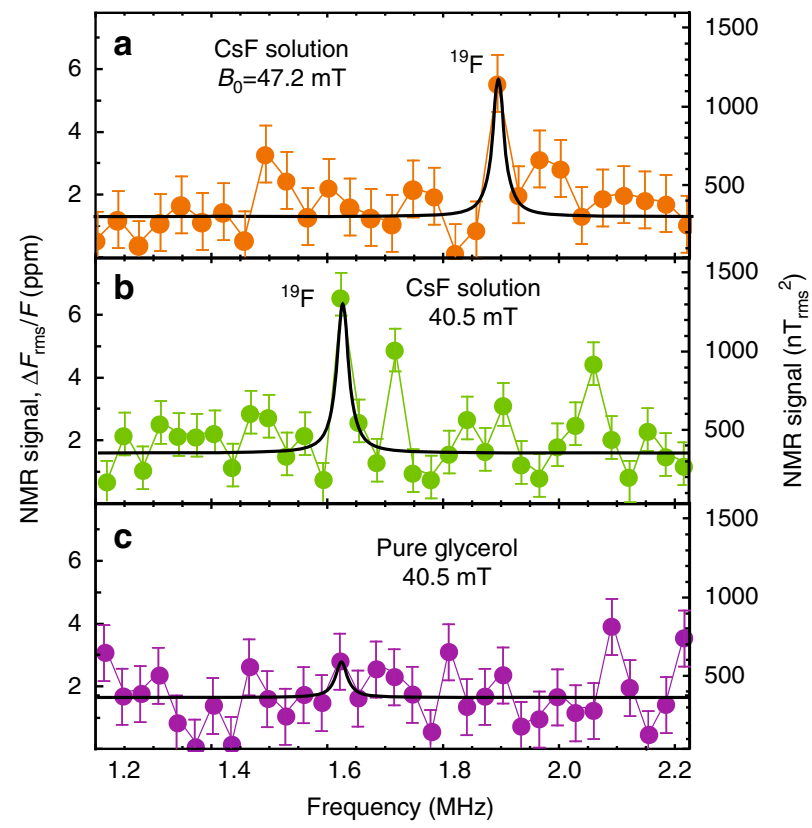

Fig. 5 Solution NMR. a, b NMR spectra of $20 \%$ by weight CsF solution in glycerol at 47.2 and $40.5 \mathrm{mT}$. c NMR spectrum of pure glycerol. All spectra were measured with an XY8-10 correlation spectroscopy pulse sequence tuned to the ${ }^{19} \mathrm{~F}$ precession frequency. Each spectrum was fit to a single Lorentzian function with central frequency set to the anticipated ${ }^{19} \mathrm{~F}$ Larmor frequency. The linewidth was fixed to that of the ${ }^{1} \mathrm{H}$ linewidth in glycerol obtained under similar conditions. The only free fit parameters were peak amplitude and offset. We assume that each spectrum contains at most one NMR line (from ${ }^{19} \mathrm{~F}$ ) and other fluctuations are due to noise. Error bars represent standard deviation of off-resonant points

Figure $3 \mathrm{~b}, \mathrm{c}$ shows the time-domain and frequency-domain NMR signals for ${ }^{19} \mathrm{~F}$ nuclei in Fomblin ${ }^{\otimes}$ oil $(6600 \mathrm{Da})$ using the $20 \mathrm{keV}$ nanograting chip. The sensor response was converted to absolute units of $\mathrm{nT}^{2}$ using analytical expressions (see Supplementary Note 1) that were validated using calibrated magnetic fields from a test coil. To confirm that the signals arise from the target nuclei, we repeated these measurements at different magnetic fields and with ${ }^{1} \mathrm{H}$-rich analytes (glycerol and immersion oil). The resulting NMR peaks were always at the expected Larmor frequency of each species (Fig. 3d), and NMR peaks were either absent (for ${ }^{19} \mathrm{~F}$ ) or greatly diminished $\left(\right.$ for ${ }^{1} \mathrm{H}$ ) when the analyte was removed ${ }^{13}, 14$.

To characterize the spin-concentration sensitivity, we continuously acquired NMR spectra of ${ }^{19} \mathrm{~F}$ nuclei in Fomblin ${ }^{\oplus}$ oil and compared SNR for both flat and nanograting sensors implanted at $20 \mathrm{keV}$. Figure 4a shows characteristic NMR spectra for each. The nanograting-sensor noise, defined as the standard deviation of points adjacent to the NMR peak, is a factor of 6 smaller than the flat sensor noise. This is primarily due to the $\sim 20 \times$ larger fluorescence intensity (Fig. 2e) exhibited by the nanograting sensor, which leads to smaller relative photon shot noise. However, the nanograting sensor signal strength, defined as the ${ }^{19} \mathrm{~F}$ NMR peak amplitude in $\mathrm{nT}^{2}$, is $\sim 2.5 \times$ smaller. This was unexpected; the signal amplitude should only depend on the NV depth, which should be the same for both sensors under identical implantation conditions (Fig. 2d, inset). A likely cause for this discrepancy is that the tops of the nanogratings were inadvertently implanted because of degradation of the etch mask (Fig. 2a). Ions bombarding the nanograting tops are nearly normally incident to the surface, resulting in $\mathrm{NV}$ centers that are too deep to sense external nuclei, reducing the overall NMR signal contrast. If $50 \%$ of the $\mathrm{NV}$ centers were formed from deep implantation into the nanograting tops, we would expect a twofold reduction in signal. Other contributions may be from deep implantation into the flat bottom of the nanogratings or imperfect wetting.

Regardless, the nanograting sensors had better overall SNR. To acquire each spectrum, $\tilde{\tau}$ was swept and the signal was averaged for a variable time $t_{\mathrm{avg}}$. Figure $4 \mathrm{~b}$ plots the SNR as a function of $t_{\mathrm{avg}}$, revealing a $2.4 \times$ SNR improvement with the nanogratings. Fomblin ${ }^{\circledR}$ has $40 \pm 2 \times 10^{24}{ }^{19} \mathrm{~F}$ spins per liter, and we detect it with $\mathrm{SNR}=11.4 \pm 0.2$ at $t_{\mathrm{avg}}=1 \mathrm{~s}$. We thus determine a minimum detectable concentration $\rho_{\min }=11 \pm 1 \times$ $10^{24}$ spins per liter. Throughout we use $t_{\text {avg }}$ as the effective integration time, since the number of points used to generate the spectrum may be reduced using optimized sampling strategies $^{34}$.

Next, we performed NMR spectroscopy on more dilute solutions to demonstrate our capability. We selected CsF dissolved in glycerol as our test analyte. Glycerol was chosen as the solvent because of its high viscosity, which limits molecular diffusion, while CsF was selected as the target molecule because of its high solubility in glycerol and because ${ }^{19} \mathrm{~F}$ has the second largest magnetic moment among common nuclear isotopes. Protons have a larger magnetic moment but were not suitable because a background proton signal was often present ${ }^{13,14}$. Using a $20 \% \mathrm{CsF} /$ glycerol solution by weight, the ${ }^{19} \mathrm{~F}$ concentration is $1.0 \times 10^{24}$ spins per liter, $\sim 40$ times lower than Fomblin ${ }^{\circledast}$. To successfully obtain a spectrum with the same SNR requires $\sim 1600$ times more signal averaging, a task previously not practical in $\mathrm{NV}$ NMR.

Figure 5 shows NMR spectra for this solution. At $B_{0}=47.2 \mathrm{mT}$, we observe a peak at the ${ }^{19} \mathrm{~F}$ Larmor frequency. When changing 


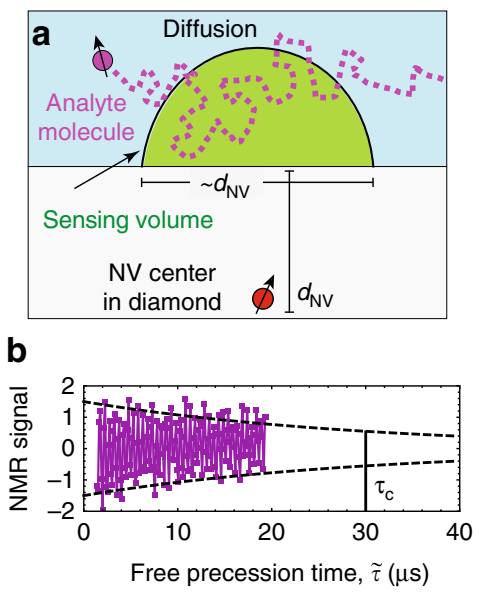

c

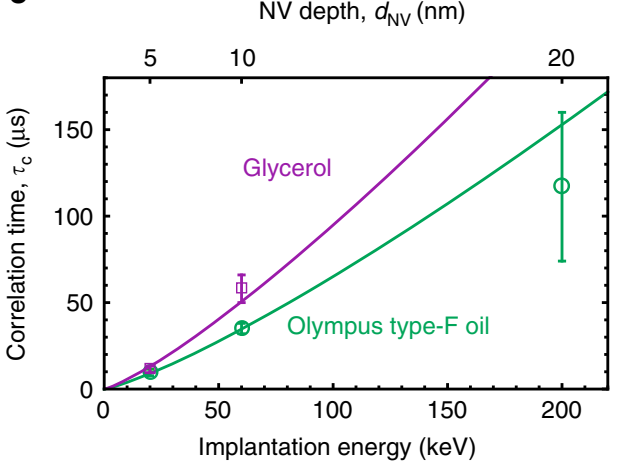

Fig. 6 Diffusion-limited NMR. a Diffusion of analyte molecules through the sensing volume. b Example of temporal decay of the correlation signal for immersion oil. The decay is exponential with a characteristic correlation time $\tau_{\mathrm{C}}$. Dashed line represents the decay envelope. c Correlation time $\tau_{\mathrm{C}}$ as a function of nitrogen implantation energy for immersion oil and glycerol. Error bars represent fit uncertainty. Solid lines are fits to the one-sided diffusion model discussed in the text

the magnetic field to $B_{0}=40.5 \mathrm{mT}$, the peak moves according to the ${ }^{19} \mathrm{~F}$ gyromagnetic ratio and maintains a comparable amplitude. Finally, when the analyte is replaced with pure glycerol the peak disappears, as expected. For the ${ }^{19} \mathrm{~F}$ peak, the SNR is $4 \pm 1$ at $t_{\mathrm{avg}}=77 \mathrm{~s}$, which corresponds to a minimum detectable concentration $\rho_{\min }=6 \pm 2 \times 10^{24}$ spins per liter. This is roughly a factor of 2 better than for Fomblin ${ }^{\circledR}$ measurements due to more optimal readout timing.

\section{Discussion}

The present platform still has significant room for improvement in sensitivity and spectral resolution. The observed sensitivity is about two orders of magnitude worse than the theoretical sensitivity calculated with Eq. (1) and plotted in Fig. 1a. The largest contributions to this discrepancy are (see Supplementary Note 1): NV control pulse errors ${ }^{35}$, which reduce the contrast by $\sim 5 \times$ from the ideal case; imperfect doping parameters, which reduce $N_{\mathrm{NV}}$ by up to $5 \times$ and increase $d_{\mathrm{NV}}$ by up to $2 \times$ from the ideal case; and the previously discussed $\sim 2.5 \times$ lower signal strength for nanograting samples. The spectral resolution, which is inversely proportional to the analyte correlation time $\tau_{\mathrm{C}}$, is currently limited to a few $\mathrm{kHz}$. For nanoscale sensing volumes, analyte molecules rapidly diffuse across the sensing region (Fig. 6a), limiting $\tau_{\mathrm{C}}$. Figure $6 \mathrm{~b}$ plots a typical correlation signal that decays exponentially with time constant, $\tau_{\mathrm{C}}$, in the microsecond range. Figure $6 \mathrm{c}$ plots $\tau_{\mathrm{C}}$ of protons in immersion oil and glycerol as a function of NV layer depth $d_{\mathrm{NV}}$. The data are fit with a one-sided diffusion model ${ }^{36}, \tau_{\mathrm{C}}=2 d_{\mathrm{NV}}^{2} / D$, where $D$ is the molecular diffusion coefficient, revealing $D_{\text {glyc }}=4.4 \pm 0.6 \times 10^{-12}$ $\mathrm{m}^{2} / \mathrm{s}$ and $D_{\text {oil }}=6.4 \pm 0.3 \times 10^{-12} \mathrm{~m}^{2} / \mathrm{s}$. These values are slightly higher than room-temperature literature values $\left(D_{\text {glyc }}=\right.$ $2.5 \times 10^{-12} \mathrm{~m}^{2} / \mathrm{s}$ and $\left.D_{\text {oil }}=0.5-2.5 \times 10^{-12} \mathrm{~m}^{2} / \mathrm{s}\right)^{36,37,38}$, which may be attributed to elevated analyte temperature or hygroscopic effects. The qualitative agreement supports the hypothesis that diffusion is responsible for the short correlation times and improves confidence in the NV depths reported by simulations. Lowering the temperature or using microporous media ${ }^{39}$ would restrict translational diffusion, improving the resolution and $\rho_{\text {min }}$, although dipolar broadening may be a limitation. Alternatively, the use of dynamic nuclear polarization may enable NV NMR with nuclear- $T_{1}$-limited resolution and improved sensitivity due to coherent nuclear precession ${ }^{40,41}$.

Nevertheless, the current platform's record sensitivity makes it promising for solid-state NMR and nuclear quadrupole resonance spectroscopy of trace powders and thin films ${ }^{42}$, 43 , since these applications require only coarse frequency resolution and diffusion is restricted. It may also find application in NMR relaxometry $^{44}$ and solution NMR for impurity profiling and quality control of pharmaceuticals ${ }^{45}$. These applications could benefit from the ease of microfluidic integration, which would permit parallel measurements. Future implementations may also harness the optical waveguiding properties of the nanogratings to reduce excitation intensity and increase fluorescence collection by exciting and collecting light through the sides of the chip.

In summary, we performed NMR spectroscopy of picoliter-volume solutions using a nanostructured diamond-chip platform. The sensor uses non-inductive detection of statistical polarization, avoiding the need for large magnetic fields or hyperpolarization. Etching dense, high-aspect-ratio nanogratings into the diamond surface resulted in a 15-fold improvement in surface area and more than 20-fold increase in fluorescence intensity without sacrificing the $\mathrm{NV}$ spin properties. Using a solution of CsF in glycerol, we determined $4 \pm 2 \times 10^{12}$ spins in an $\sim 1 \mathrm{pL}$ volume can be detected with a SNR of 3 in $1 \mathrm{~s}$ integration. This concentration sensitivity was independently confirmed using Fomblin oil and found to agree to within a factor of 2. This represents nearly two orders of magnitude improvement over previous picoliter NMR demonstrations.

\section{Methods}

Fabrication details. Nanogratings were fabricated on the surfaces of electronic-grade diamond chips purchased from Element 6 (initial dimensions $2 \times 2 \times 0.5 \mathrm{~mm}^{3}, 1.1 \%{ }^{13} \mathrm{C}$ abundance, [100]-polished faces, [110] sides). After cleaning the chips for $7 \mathrm{~h}$ in a 1:1:1.3 mixture of nitric:perchloric:sulfuric acids at $200{ }^{\circ} \mathrm{C}$ (from now on referred to as triacid cleaning), each diamond chip was mounted flat on a silicon substrate. The chip was surrounded by four 0.5 -mm-thick stainless-steel sheets flush with the diamond surface to avoid edge beading during spin coating. A thin film of i-CON 16 was used as an adhesive to stick the diamond and the steel sheets to the silicon substrate because it does not outgas or reflow during baking. The next step was to spin-coat i-CON 16 as an anti-reflection coating (ARC) on top of the diamond ( $4000 \mathrm{rpm}, 150{ }^{\circ} \mathrm{C}$ oven bake for $5 \mathrm{~min}$ ), followed by spin-coating with a ultraviolet (UV) negative photoresist (NR-7 500p, $4000 \mathrm{rpm}, 150^{\circ} \mathrm{C}$ hot plate bake for $1 \mathrm{~min}$ ).

Interferometric lithography ${ }^{26,27}$ was used to produce optical standing waves with a period of $\sim 400 \mathrm{~nm}$. With interferometric lithography, the entire diamond surface was covered with periodic nanostructures in a few seconds of exposure time. By varying the resist, postbake, and development conditions, the grating duty cycle can span at least $20-80 \%$. Most of the samples used in this work had $\sim 50 \%$ duty cycle. After developing for $1 \mathrm{~min}$ in RD6 developer, the un-masked regions of ARC were removed using a reactive ion etch $(10 \mathrm{sccm} \mathrm{O}$ flow, $10 \mathrm{~W}$ radio frequency power) for $1 \mathrm{~min}$. For the metal mask, 3-5 $\mathrm{nm}$ of $\mathrm{Cr}$ (used for adhesion) followed by $70 \mathrm{~nm}$ of gold were deposited on the masked diamond chips. Liftoff 
was performed by immersing the chips in a sonicating acetone bath for $10 \mathrm{~min}$ to leave only $\mathrm{Cr} / \mathrm{Au}$ grating structures on the diamond surface. Next, a highly anisotropic oxygen/argon etch using inductively coupled plasma (ICP; $8 \mathrm{sccm} \mathrm{Ar}_{2}$ flow, $16 \mathrm{sccm} \mathrm{\textrm {O } _ { 2 }}$ flow, $30 \mathrm{~W}$ forward radio frequency power, $450 \mathrm{~W}$ ICP radio frequency power) was applied for $40 \mathrm{~min}$ to form nanogratings up to $3 \mu \mathrm{m}$ deep.

In the final etch step, there is a tradeoff between etching deeper and losing the mask. Ideally, the mask would remain thick enough ( $\gtrsim 10 \mathrm{~nm})$ to block ions from penetrating the tops of the nanogratings during implantation. This is because ions incident on the nanograting tops would travel on average approximately six times deeper than those incident to the sidewalls due to the large difference in angles of incidence. At such a large depth these NV centers will hardly register any NMR signal, reducing the overall NMR signal contrast. We found that the $40 \mathrm{~min}$ of etching used in this work was longer than optimal, and the mask had eroded over large regions of the chips prior to implantation. We have since optimized the process to use a slightly thicker metal mask such that the mask remains largely intact after 35 min of etching.

After fabrication, the nanograting sidewalls were implanted with nitrogen. When possible, we left the remaining metal mask used for etching to block ions from penetrating deep into the tops of the gratings. Implantation was performed by Materials Diagnostics (Albany, NY) using a ${ }^{15} \mathrm{~N}^{+}$ion beam with $20-200 \mathrm{keV}$ implantation energy (corresponding to $\sim 5-20 \mathrm{~nm}$ typical NV depth), delivering a dose of $2 \times 10^{13}$ to $8 \times 10^{13}{ }^{15} \mathrm{~N}^{+}$ions $/ \mathrm{cm}^{2}$ at 4 degree implantation angles (Supplementary Note 2 ). For comparison, flat chips were mounted vertically (to implant at the same angle; see Fig. 2d, inset). Following implantation, the chips were cleaned in triacid overnight, then annealed in vacuum in a multistep annealing process $\left(800^{\circ} \mathrm{C}\right.$ for $4 \mathrm{~h}$ followed by $1100^{\circ} \mathrm{C}$ for $2 \mathrm{~h}$ ) to form an NV layer at the nanograting sidewall surfaces. This annealing procedure was selected to give a high $\mathrm{NV}^{-}$yield while minimizing the abundance of other paramagnetic impurities ${ }^{46}$. After annealing, the diamond chips were again cleaned in triacid for $10 \mathrm{~h}$ to remove surface residues and mounted in the microscope set-up. Triacid treatment is expected to yield a hydrophilic, oxygen-terminated surface. Although the triacid treatment proved sufficient for wetting and NV properties, other treatments may provide additional advantages ${ }^{47-49}$. Each time we removed a diamond chip from the set-up, we used the same acid-washing procedure to remove immersion oil, Fomblin oil, CsF/glycerol solutions, or other contaminations.

Experimental procedure. After mounting a diamond chip in the microscope, we align the $B_{0}$ field from a permanent magnet by measuring the optically detected magnetic resonance (ODMR) frequencies of the four NV orientations. Good field alignment is important because $T_{2}$ is maximized and the $\mathrm{NV}$ contrast is maximized when $B_{0}$ is aligned along the $\mathrm{N}-\mathrm{V}$ axis ${ }^{50}$, resulting in better sensitivity. We use the NV sub-ensemble aligned with $B_{0}$ for correlation spectroscopy, while the other three NV sub-ensembles do not participate in the measurement and contribute background fluorescence. After aligning, we calculate the $B_{0}$ magnitude from the ODMR spectrum. We position the microwave wire to achieve a reasonably fast Rabi frequency $(10-15 \mathrm{MHz})$ and test the correlation spectroscopy experiment with the AC magnetic field from a calibrated test coil (driven by a sine wave from a function generator).

When performing NMR spectroscopy, we select parameters for the correlation pulse sequence based on the following principles. Laser light pulses have $5 \mu \mathrm{s}$ duration. The first $\sim 1 \mu$ s is used for readout, while the remainder is used to efficiently repolarize the NV centers. The separation between $\pi$-pulses, $2 \tau$, is chosen to match half the nuclear Larmor period of the target spin, $4 \tau=\tau_{\mathrm{L}}$. We select the number of repetitions (the " $N$ " in XY8-N) which produces the highest signal-to-noise-ratio (SNR), at constant measurement time, using protons in pure glycerol as a convenient test sample. The step size between $\tilde{\tau}$ values is chosen to be $\sim \tau_{\mathrm{L}} / 4$, and the longest value is chosen to match the approximate duration of the XY8-N sequences. This represents a compromise between obtaining high SNR (which would seek to minimize the overall measurement time) and obtaining high spectral resolution (which would prefer to use as long a $\tilde{\tau}$ as possible). In every experiment we alternate the phase of the final $\pi / 2$-pulse and subtract the fluorescence signals, resulting in fast common-mode rejection of fluorescence intensity drifts.

We have noticed that the fluorescence intensity depends on the time between laser pulses in a non-trivial manner, presumably due to complex $\mathrm{NV}^{0} / \mathrm{NV}^{-}$ dynamics. To circumvent related systematic effects, we add buffer time between the last microwave $\pi / 2$-pulse and the laser readout pulse to ensure that the time between laser pulses remains constant while we sweep $\tilde{\tau}$. However, this means most experiments take $\sim 1.5 \times$ longer than they should because of the buffer time. We average fluorescence readout time traces on an oscilloscope, which introduces additional dead time. The oscilloscope misses triggers while averaging and data processing, and it also spends time transferring averaged time traces to the acquisition computer. These dead times collectively make the actual experiments roughly $\sim 2.5 \times$ slower than necessary, which we will improve in future setups. When determining $t_{\text {avg }}$ in the main text, we neglect this dead time.

Data availability. The data used to generate the figures in this work are available from the corresponding author upon request.
Received: 10 February 2017 Accepted: 15 June 2017

Published online: 04 August 2017

\section{References}

1. Lee, H., Sun, E., Ham, D. \& Weissleder, R. Chip-NMR biosensor for detection and molecular analysis of cells. Nat. Med. 14, 869-874 (2008).

2. Ha, D., Paulsen, J., Sun, N., Song, Y.-Q. \& Ham, D. Scalable NMR spectroscopy with semiconductor chips. Proc. Natl Acad. Sci. USA 111, 11955-11960 (2014).

3. Rubakhin, S. S., Romanova, E. V., Nemes, P. \& Sweedler, J. V. Profiling metabolites and peptides in single cells. Nat. Methods 8, S20-S29 (2011).

4. Fratila, R. M. \& Velders, A. H. Small-volume nuclear magnetic resonance spectroscopy. Annu. Rev. Anal. Chem. 4, 227-249 (2011).

5. Lacey, M. E., Subramanian, R., Olson, D. L., Webband, A. G. \& Sweedler, J. V. High-resolution NMR spectroscopy of sample volumes from $1 \mathrm{~nL}$ to $10 \mu \mathrm{L}$. Chem. Rev. 99, 3133-3152 (1999).

6. Olson, D. L., Peck, T. L., Webb, A. G., Magin, R. L. \& Sweedler, J. V. High-resolution microcoil 1H-NMR for mass-limited, nanoliter-volume samples. Science 270, 1967-1970 (1995).

7. Krojanski, H. G., Lambert, J., Gerikalan, Y., Suter, D. \& Hergenroder, R. Microslot NMR probe for metabolomics studies. Anal. Chem. 80, 8668-8672 (2008).

8. McDowell, A. F. \& Adolphi, N. L. Operating nanoliter scale NMR microcoils in a 1 tesla field. J. Magn. Reson. 188, 74-82 (2007)

9. Ledbetter, M. P. \& Budker, D. Zero-field nuclear magnetic resonance. Phys. Today 66, 44-49 (2013).

10. Clarke, J., Hatridge, M. \& Mößle, M. SQUID-detected magnetic resonance imaging in microtesla fields. Annu. Rev. Biomed. Eng. 9, 389-413 (2007).

11. Staudacher, T. et al. Nuclear magnetic resonance spectroscopy on a (5-nanometer) sample volume. Science 339, 561-563 (2013).

12. Mamin, H. J. et al. Nanoscale nuclear magnetic resonance with a nitrogen-vacancy spin sensor. Science 339, 557-560 (2013).

13. DeVience, S. J. et al. Nanoscale NMR spectroscopy and imaging of multiple nuclear species. Nat. Nanotechnol. 10, 129-134 (2015).

14. Rugar, D. et al. Proton magnetic resonance imaging using a nitrogenvacancy spin sensor. Nat. Nanotechnol. 10, 120-124 (2015).

15. Häberle, T., Schmid-Lorch, D., Reinhard, F. \& Wrachtrup, J. Nanoscale nuclear magnetic imaging with chemical contrast. Nat. Nanotechnol. 10, 125-128 (2015).

16. Loretz, M. et al. Spurious harmonic response of multipulse quantum sensing sequences. Phys. Rev. X 5, 021009 (2015).

17. Staudacher, T. et al. Probing molecular dynamics at the nanoscale via an individual paramagnetic centre. Nat. Commun. 6, 8527 (2015).

18. Lovchinsky, I. et al. Nuclear magnetic resonance detection and spectroscopy of single proteins using quantum logic. Science 351, 836-841 (2016).

19. Herzog, B. E., Cadeddu, D., Xue, F., Peddibhotla, P. \& Poggio, M. Boundary between the thermal and statistical polarization regimes in a nuclear spin ensemble. Appl. Phys. Lett. 105, 043112 (2014).

20. Hashi, K. et al. Achievement of 1020 MHz NMR. J. Magn. Reson. 256, 30-33 (2015).

21. Polenova, T. \& Budinger, T. F. Ultrahigh field NMR and MRI: Science at a crossroads. Report on a jointly-funded NSF, NIH and DOE workshop, held on November 12-13, 2015 in Bethesda, Maryland, USA. J. Magn. Reson. 266, 81-86 (2016)

22. Kovacs, H., Moskau, D. \& Spraul, M. Cryogenically cooled probes-a leap in NMR technology. Progr. Nucl. Magn. Reson. Spectrosc. 46, 131-155 (2005).

23. Guitard, P. A. et al. Local nuclear magnetic resonance spectroscopy with giant magnetic resistance-based sensors. Appl. Phys. Lett. 108, 212405 (2016).

24. Verpillat, F. et al. Remote detection of nuclear magnetic resonance with an anisotropic magnetoresistive sensor. Proc. Natl Acad. Sci. USA 105, 2271-2273 (2008).

25. Ledbetter, M. P. et al. Zero-field remote detection of NMR with a microfabricated atomic magnetometer. Proc. Natl Acad. Sci. USA 105 , 2286-2290 (2008).

26. Brueck, S. R. J. Optical and interferometric lithography-nanotechnology enablers. Proc. IEEE 93, 1704-1721 (2005).

27. Xia, D., Ku, Z., Lee, S. C. \& Brueck, S. R. J. Nanostructures and functional materials fabricated by interferometric lithography. Adv. Mater. 23, 147-179 (2011).

28. Faraon, A. et al. Quantum photonic devices in single-crystal diamond. New J. Phys. 15, 025010 (2013).

29. Hausmann, B. J. M. et al. Fabrication of diamond nanowires for quantum information processing applications. Diamond Relat. Mater. 19, 621-629 (2010).

30. Acosta, V. M. et al. Diamonds with a high density of nitrogen-vacancy centers for magnetometry applications. Phys. Rev. B 80, 115202 (2009). 
31. de Lange, G., Wang, Z. H., Risté, D., Dobrovitski, V. V. \& Hanson, R. Universal dynamical decoupling of a single solid-state spin from a spin bath. Science 330, 60-63 (2010).

32. Rondin, L. et al. Magnetometry with nitrogen-vacancy defects in diamond. Rep. Progr. Phys. 77, 056503 (2014).

33. Laraoui, A. et al. High-resolution correlation spectroscopy of ${ }^{13} \mathrm{C}$ spins near a nitrogen-vacancy centre in diamond. Nat. Commun. 4, 1651 (2013).

34. Scheuer, J. et al. Accelerated 2D magnetic resonance spectroscopy of single spins using matrix completion. Sci. Rep. 5, 17728 (2015).

35. Farfurnik, D. et al. Optimizing a dynamical decoupling protocol for solid-state electronic spin ensembles in diamond. Phys. Rev. B 92, 060301 (2015).

36. Pham, L. M. et al. NMR technique for determining the depth of shallow nitrogen-vacancy centers in diamond. Phys. Rev. B 93, 045425 (2016).

37. Tomlinson, D. J. Temperature dependent self-diffusion coefficient measurements of glycerol by the pulsed N.M.R. technique. Mol. Phys. 25, 735-738 (1973).

38. Loretz, M., Pezzagna, S., Meijer, J. \& Degen, C. L. Nanoscale nuclear magnetic resonance with a 1.9-nmdeep nitrogen-vacancy sensor. Appl. Phys. Lett. 104, 033102 (2014)

39. Li, H., Eddaoudi, M., O’Keeffe, M. \& Yaghi, O. M. Design and synthesis of an exceptionally stable and highly porous metal-organic framework. Nature 402, 276-279 (1999).

40. Abrams, D., Trusheim, M. E., Englund, D. R., Shattuck, M. D. \& Meriles, C. A. dynamic nuclear spin polarization of liquids and gases in contact with nanostructured diamond. Nano Lett. 14, 2471-2478 (2014).

41. London, P. et al. Detecting and polarizing nuclear spins with double resonance on a single electron spin. Phys. Rev. Lett. 111, 067601 (2013).

42. Yesinowski, J. P., Buess, M. L., Garroway, A. N., Ziegeweid, M. \& Pines, A. Detection of $14 \mathrm{~N}$ and $35 \mathrm{Cl}$ in cocaine base and hydrochloride using NQR, NMR, and SQUID techniques. Anal. Chem. 67, 2256-2263 (1995).

43. Garroway, A. N. et al. Remote sensing by nuclear quadrupole resonance. IEEE Trans. Geosci.Remote Sensing 39, 1108-1118 (2001).

44. Demas, V. \& Lowery, T. J. Magnetic resonance for in vitro medical diagnostics: superparamagnetic nanoparticle-based magnetic relaxation switches. New J. Phys. 13, 025005 (2011).

45. Maggio, R. M., Calvo, N. L., Vignaduzzo, S. E. \& Kaufman, T. S. Pharmaceutical impurities and degradation products: uses and applications of NMR techniques. J. Pharm. Biomed. Anal. 101, 102-122 (2014).

46. Yamamoto, T. et al. Extending spin coherence times of diamond qubits by high-temperature annealing. Phys. Rev. B 88, 075206 (2013).

47. Hauf, M. V. et al. Chemical control of the charge state of nitrogen-vacancy centers in diamond. Phys. Rev. B 83, 081304 (2011).

48. Cui, S. \& Hu, E. L. Increased negatively charged nitrogen-vacancy centers in uorinated diamond. Appl. Phys. Lett. 103, 051603 (2013).

49. Yamano, H. et al. Charge state stabilization of shallow nitrogen vacancy centers in diamond by oxygen surface modification. Jpn. J. Appl. Phys. 56, 04CK08 (2017).

50. Stanwix, P. L. et al. Coherence of nitrogen-vacancy electronic spin ensembles in diamond. Phys. Rev. B 82, 201201 (2010).

51. Stone, N. J. Table of Nuclear Magnetic Dipole and Electric Quadrupole Moments. Report No. INDC(NDS)-0658 (IAEA Vienna, INDC International Nuclear Data Committee, 2014).

\section{Acknowledgements}

We gratefully acknowledge advice and support from C. Santori, Z. Huang, D. Twitchen, D. Suter, D. Bucher, D. Glenn, R. Walsworth, F. Hubert, Y. Silani, the UNM Comprehensive Cancer Center Fluorescence Microscopy Shared Resource, and the DOE Center for Integrated Nanotechnologies (CINT). This work was made possible by funding from NSF (IIP \#1549836) and NIH (1R41GM119925-01). P.K. acknowledges support from the Intelligence Community Postdoctoral Research Fellowship Program. L. B. is supported by a Marie Curie Individual Fellowship within the second Horizon 2020 Work Programme. J.S. acknowledges support from the Baltic American Freedom Foundation

\section{Author contributions}

A.J., D.B., and V.M.A. conceived the idea. P.K., A.J., and V.M.A. planned and carried out the experiments and analysis in V.M.A.'s lab at UNM. N.M., I.F., and F.M.B. fabricated and characterized the nanograting sensors under supervision of A.N., S.R.J.B., and V.M.A. F.M.B. assembled the optical NMR apparatus and P.K. developed the software automation. A.L., J.S., and L.B. contributed to data collection and analysis. All authors discussed results and contributed to the writing of the manuscript.

\section{Additional information}

Supplementary Information accompanies this paper at doi:10.1038/s41467-017-00266-4.

Competing interests: A.J., D.B., and L.B. are co-founders of startup ODMR Technologies and have financial interests in the company. The remaining authors declare no competing financial interests.

Reprints and permission information is available online at http://npg.nature.com/ reprintsandpermissions/

Publisher's note: Springer Nature remains neutral with regard to jurisdictional claims in published maps and institutional affiliations.

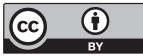

Open Access This article is licensed under a Creative Commons Attribution 4.0 International License, which permits use, sharing, adaptation, distribution and reproduction in any medium or format, as long as you give appropriate credit to the original author(s) and the source, provide a link to the Creative Commons license, and indicate if changes were made. The images or other third party material in this article are included in the article's Creative Commons license, unless indicated otherwise in a credit line to the material. If material is not included in the article's Creative Commons license and your intended use is not permitted by statutory regulation or exceeds the permitted use, you will need to obtain permission directly from the copyright holder. To view a copy of this license, visit http://creativecommons.org/ licenses/by/4.0/

(c) The Author(s) 2017 